\title{
Lo mejor de uno mismo y las alternativas epistémicas*
}

\author{
Doing One's Best and the Epistemic alternatives
}

TOBIES GRIMALTOS ${ }^{* *}$

\begin{abstract}
Resumen: El Principio de Posibilidades Alternativas (PPA) supone un problema para las posiciones compatibilistas respecto de la responsabilidad moral. Desde que Harry Frankfurt diseñara uno, los (supuestos) contraejemplos al PPA se conocen como casos Frankfurt. Una de las mejores líneas de defensa de este principio frente a los nuevos casos Frankfurt es la que se ofrece en Moya (2011 y 2014). En este artículo me propongo mostrar que su estrategia no es satisfactoria y, al mismo tiempo, argumentar que para la atribución de responsabilidad moral por una acción es suficiente (ceteris paribus) con que existan alternativas epistémicas. Palabras Clave: Casos Frankfurt, alternativas robustas, responsabilidad moral, alternativas epistémicas.
\end{abstract}

\begin{abstract}
The Principle of Alternate Possibilities (PAP) poses a problem for compatibilist positions regarding moral responsibility. Since Harry Frankfurt designed one, the counter-examples to the PAP are known as Frankfurt cases. One of the best ways to defend PAP against the new Frankfurt cases is the one offered in Moya (2011 and 2014). In this article I intend to show that his strategy is not satisfactory and, at the same time, to argue that for the attribution of moral responsibility for an action it is sufficient (ceteris paribus) that there are epistemic alternatives.
\end{abstract}

Keywords: Frankfurt cases, robust alternatives, moral responsibility, epistemic alternatives.

\section{Introducción}

En opinión de muchos teóricos, una de las condiciones necesarias para ser moralmente responsable por una acción es disponer de alternativas: poder realizar dicha acción o abstenerse de hacerla. En esto consiste el llamado Principio de Posibilidades Alternativas (PPA): un sujeto es moralmente responsable de su decisión y de su acción consiguiente sólo si hubiera podido decidir o actuar de otro modo (o al menos si hubiera podido decidir evitar actuar del modo que lo hizo). El PPA supone un problema para las posiciones compatibilistas respecto de la responsabilidad moral: las posiciones que defienden que la responsabilidad moral y determinismo son compatibles. El problema (se dice) es que si el determinismo es verdadero, entonces, en

Recibido: 02/01/2018. Aceptado: 14/05/2018.

* La investigación conducente a este trabajo ha sido financiada por el Ministerio de Economía y Competitividad y el Fondo Europeo de Desarrollo Regional como parte del proyecto “Autoconocimiento, responsabilidad moral y autenticidad”, FFI2016-75323-P. Agradezco la ayuda y estímulo prestados. Agradezco asimismo los valiosos comentarios de dos revisores anónimos a una versión anterior de este trabajo.

** Catedrático del Departamento de Filosofía de la Universitat de València. Correo electrónico: tobies.grimaltos@ uv.es. Principales líneas de investigación: Teoría del conocimiento, testimonio y justificación, mentira y engaño. Artículos recientes: "More and More Lies. A New Distintion and Its Consequences", Teorema, XXXVI/1, 2017: 5-21 con Sergi Rosell; "La mentida, l'engany i la seua valoració moral. Una defensa (parcial) de la mentida", Comprendre, 19/1, 2017: 5-18. 
realidad no existen alternativas, nadie podría actuar realmente de modo distinto a como lo hace en cada caso. Si el PPA es correcto y si el determinismo es verdadero, no existe responsbilidad moral, porque el determinismo implica que no existan posibilidades alternativas.

Harry Frankfurt (1969) diseñó un caso destinado a mostrar que el PPA es falso, esto es, un contraejemplo al PPA. Se trata de un caso en el que supuestamemente un sujeto es moralmente responsable (culpable) de su acción a pesar de no disponer de alternativa (de no poder actuar de otro modo a como lo hizo) ${ }^{1}$. Desde entonces, los (supuestos) contraejemplos al PPA se conocen como casos Frankfurt y han experimentado una sofistificación creciente ante las distintas respuestas de los defensores del PPA. Una de las mejores líneas de defensa de este principio frente a los nuevos casos Frankfurt es la que ofrece Carlos Moya en "On the Very Idea of a Robust Alternative" (Moya 2011) y "Doing One's Best, Alternative Possibilities and Blameworthiness" (Moya 2014). Los casos Frankfurt más potentes en la actualidad son los diseñados por Derk Pereboom y David Widerker (Pereboom 2009 y Widerker 2009) y contra ellos justamente construye Moya su defensa. La idea central de Moya es que una consideración atenta de lo que constituye una alternativa "robusta" muestra que tal tipo de contraejemplos fracasan, pues o bien no consiguen eliminar las alternativas robustas por parte del agente o bien no satisfacen algún otro requisito necesario para que el caso sea realmente un contraejemplo al PPA. En este artículo me ocuparé sólo de la respuesta de Moya al caso de Pereboom, puesto que bastará con mostrar que su estrategia no consigue arruinarlo para probar que no es satisfactoria contra los casos tipo Frankfurt y que, por lo tanto, no es una defensa exitosa del PPA.

Al mismo tiempo que muestro la falta de éxito de Moya en su defensa del PPA, argumentaré que (ceteris paribus) ${ }^{2}$ es suficiente con que existan alternativas epistémicas ${ }^{3}$ para la atribución de responsabilidad moral por una acción. Un sujeto es moralmente responsable de su acción si cree que tiene alternativa ${ }^{4}$. Esto es, si el PPA mantiene que un sujeto es moralmente responsable de su decisión y de su acción consiguiente sólo si hubiera podido decidir o actuar de otro modo (o al menos si hubiera podido decidir evitar actuar del modo en que lo hizo), yo me propongo defender que un sujeto es moralmente responsable de su decisión y su acción consecuente si (dadas las otras condiciones mencionadas) cree que podría actuar de otro modo. Es decir, uno es responsable de hacer A si cree que podría no hacer $\mathrm{A}^{5}$.

1 Podemos encontrar un antecedente histórico de tal tipo de casos en Locke (1689/1956, II, XXI, 10 :

Supongamos que un hombre es llevado mientras duerme a la habitación en la que se encuentra una persona que desea ver y con la que quiere hablar; y que este hombre sea encerrado, de manera que no pueda salir. Cuando despierte, estará feliz de encontrarse con la compañía deseada con la que decidirá quedarse, es decir, que preferirá permanecer allí en lugar de salir fuera. Y yo pregunto: ¿no es esta estancia voluntaria?

Parece que tal sujeto es responsable de permanecer dentro de la habitación a pesar de no poder salir de ella.

2 Es decir, si se da además la voluntariedad y el control racional: si la acción es voluntaria y responde a las razones que tiene el agente.

3 Por alternativa epistémica entenderé aquí aquella posibilidad (acción o decisión normalmente) que el sujeto cree que puede realizar (o al menos no está convencido de que no puede realizarla) tanto si es el caso que puede hacerlo como si no.

4 Tal creencia suele ser tácita, pero el hecho de que delibere muestra que el agente no ha excluido la posibilidad de actuar de otro modo.

5 Por otro lado, probablemente también es verdad que uno no es responsable moralmente de no hacer A (ni de no decidir hacer A), aunque pudiera hacerlo, si está convencido (de manera justificada) de que no puede hacerlo. Pero ésta es otra cuestión en la que no entraremos aquí. 


\section{Nuevos casos Frankfurt no deterministas}

Un típico caso Frankfurt, podría ser el siguiente:

Supongamos que Black, un malvado neurocientífico, quiere que Jones mate a Smith. El propio Jones está deliberando de hecho sobre si matarlo o no. Black quiere asegurarse de que lo hace y a tal efecto ha implantado en el cerebro de Jones, sin que éste sea consciente de ello, un dispositivo que permite que Black observe los procesos mentales que tienen lugar durante la deliberación de Jones. Tal dispositivo permite también que Black pueda causar la decisión de Jones de matar a Smith, aunque prefiere, si es posible, no actuar. Black quiere asegurarse de que Jones mate a Smith, pero prefiere no intervenir si no es necesario. Si Black observara que Jones está a punto de decidir no matar a Smith, entonces activará el dispositivo y causará la decisión de Jones. Sin embargo, finalmente Jones decide por sí mismo matar a Smith y así lo hace, sin que Back haya tenido que intervenir.

Algo que se ha achacado frecuentemente a este tipo de casos es su falta de concreción. Se ha dicho que habría que describir con más detalle lo que realmente acontece en ellos. Y en este sentido se les ha opuesto lo que se conoce como la Estrategia del dilema, que es a su vez una defensa del PPA, por lo que también recibe el nombre de Defensa del dilema ${ }^{6}$. Esta objeción puede formularse del siguiente modo. Black se sirve de algún signo por parte de Jones para no intervenir (quizá un tic que se produce en su rostro cuando está a punto de tomar la decisión que a Black le conviene, quizá la secreción de ciertas sustancias en su cerebro o la contemplación de ciertos pensamientos...), un signo que ha de ser previo a la decisión de Jones. Ahora bien, la cuestión es: o bien este signo es causalmente suficiente para la decisión de Jones (o está asociado a alguna condición causalmente suficiente) o no lo es, sino que es más bien un síntoma fiable, aunque no infalible, de la próxima decisión de Jones de matar a Smith. Si es causalmente suficiente, entonces la decisión de Jones está causalmente determinada y los incompatibilistas no aceptarán que Jones sea moralmente responsable, puesto que mantienen la incompatibilidad entre responsabilidad moral y determinismo. Si sólo es un signo fiable, entonces será moralmente responsable, pero no hay ninguna razón concluyente para pensar que no hubiera podido decidir finalmente de un modo distinto a como lo hizo.

La verdad es que no he acabado de ver nunca la eficacia del primer cuerno del dilema. Si los incompatibilistas no aceptarían que Jones sea moralmente responsable, puesto que mantienen la incompatibilidad entre responsabilidad moral y determinismo, entonces, me da la impresión que están prejuzgando la cuestión, ya que lo que se trata de comprobar precisamente con estos casos es si, para la responsabilidad moral, es verdaderamente necesaria una condición que (supuestamente) el determinismo haría imposible, esto es, la existencia de alternativas. Si el determinismo fuera incompatible con la responsabilidad moral, sería al menos porque implicaría que no existen alternativas ${ }^{7}$ y justamente lo que

6 Entre los autores que han usado esta estrategia están Kane (1985), Ginet (1996) y Widerker (1995), siendo quizá la de este último la más elaborada.

7 Según algunos autores, implicaría también que el agente no fuera la fuente o el origen último de su decisión, pero lo que nos interesa ahora es la supuesta incompatibilidad entre el determinismo y la existencia de posibili- 
se trata de ver con los casos Frankfurt es si la responsabilidad moral es posible a pesar de la ausencia de alternativas y, por tanto, compatible (por lo que a esto se refiere) con el determinismo. Por otro lado, que el signo determine la posterior decisión no supone tampoco comprometerse con la verdad del determinismo; los indeterministas pueden aceptar perfectamente que algunos acontecimientos determinen a otros, lo que negarán es que todos estén determinados.

En cualquier caso y, quizá también ante esta misma duda, muchos de los opositores al PPA han planteado nuevos casos Frankfurt en los que se evite el supuesto de determinismo, casos en los que el signo de que el sujeto va a decidir actuar de manera culpable no sea causa suficiente (o esté asociado a alguna condición causalmente suficiente) de tal decisión y en los que, a la vez, no exista verdaderamente una alternativa moralmente relevante, es decir, una alternativa robusta ${ }^{8}$ a su decisión.

Atendiendo, pues, a estos requisitos se han diseñado nuevos casos Frankfurt. Entre ellos, uno de los más notables es el que ofrece Derk Pereboom (Pereboom, 2009). Dice así:

Joe está considerando la posibilidad de solicitar una reducción por la tasa de registro que pagó por la compra de una casa. Sabe que reclamar dicha deducción es ilegal, pero que probablemente no lo descubrirán y que si lo hicieran podría alegar ignorancia de un modo convincente. Supongamos que tiene una fuerte tendencia, aunque no siempre predominante, de anteponer su propio interés sin tener en cuenta su coste para otros e incluso si implica alguna actividad ilegal. Además de esto, la única manera de que, en esta situación, no optara por evadir impuestos sería por la consideración de razones morales, de las que es consciente. No podría dejar de hacerlo, por ejemplo, sin ninguna razón o simplemente por capricho. Es más, es causalmente necesario para que no decida evadir impuestos en esta situación que alcance cierto nivel de atención a razones morales. Joe puede asegurar tal nivel de atención de manera voluntaria. Sin embargo el alcanzar dicho nivel de atención no es causalmente suficiente para que no opte por evadir impuestos. Si alcanzara tal nivel de atención, podría no obstante, mediante el ejercicio de su libre albedrío libertarista, o bien optar por evadir impuestos o evitar hacer tal elección [...]. Sin embargo, para asegurarse que elegirá evadir impuestos, un neurocientífico ha implantado, sin que Joe lo sepa, un dispositivo en su cerebro tal que, si detectara el nivel de atención necesario, estimularía electrónicamente los centros neurales adecuados, de tal modo que inevitablemente darían como resultado la deseada elección de Joe. Ocurre, no obstante, que Joe no alcanza tal nivel de atención a sus razones morales y elige evadir impuestos por sí mismo, mientras el dispositivo permanece inactivo" (Pereboom, 2009: 113).

Antes de continuar, analicemos con un poco de detalle este ejemplo. Parece que el sujeto es moralmente responsable de su decisión aunque no podría haber decidido actuar de otro modo, con lo cual se mostraría que la existencia de alternativas (poder actuar de otro modo) no es condición necesaria para la responsabilidad moral. Habría casos (éste al

dades alternativas.

8 Como se ha dado en denominar a estas alternativas desde que así lo hiciera Fischer (1994). 
menos) en los que un sujeto es moralmente responsable a pesar de no disponer de alternativas reales. Vemos, sin embargo, que el sujeto ignora la existencia del dispositivo en su cerebro, con lo que la alternativa de no evadir impuestos es una alternativa epistémica y meramente epistémica (para él). Que exista una alternativa meramente epistémica, obviamente no es suficiente para el defensor del PPA, pues, según él, las alternativas necesarias para la responsabilidad son las reales: no basta con que el sujeto crea que puede actuar de otro modo, ha de poder actuar realmente de otro modo. Vemos también que, aparentemente al menos, se cumple con la condición indeterminista: la atención con cierto grado de viveza a razones morales es condición necesaria, pero no suficiente, para que el sujeto decida no evadir impuestos, aunque el hecho de que no atienda vivamente a razones morales es suficiente para que decida evadirlos (Widerker 2009). Además (y por si acaso), Pereboom afirma explícitamente que el sujeto posee libre albedrío libertarista. Más aún, prestar atención a tales razones es algo que depende de su voluntad y no algo que simplemente le sucede.

Los defensores del PPA, sin embargo, objetarán a este caso que, aunque el sujeto no pueda decidir no evadir impuestos, aún posee una alternativa que está bajo su control: prestar atención con cierta intensidad a razones morales. Ahora bien, sólo si tal alternativa tiene alguna relevancia moral (si es una alternativa robusta), el PPA podrá salir indemne de un caso como éste. El problema es, pues: ¿prestar atención con cierta intensidad a razones morales es una alternativa suficientemente robusta? ¿Cuando una alternativa es verdaderamente robusta? ¿Qué condición o condiciones ha de satisfacer una alternativa para ser robusta?

\section{Alternativas robustas}

De acuerdo con Moya (Moya 2014:4), una alternativa es robusta sólo si: o bien a) el hecho de que el agente no la elija explica parcialmente su culpabilidad ${ }^{9}$ por lo que ha hecho o b) el hecho de que el agente la elija explica parcialmente porqué es menos culpable de lo que sería de otro modo, o incluso no culpable en absoluto. Cabe añadir, como advierte Moya, que tal explicación debe ser racional o justificadora y no meramente causal. Ahora bien - y ésta es su tesis principal - que una alternativa sea o no robusta es una cuestión contextual. En su opinión, los hechos modales - lo que el sujeto puede o no puede hacer en un contexto particular-, son relevantes para juzgar si una alternativa es o no robusta. Si una manera particular de actuar es o no una alternativa robusta en una situación particular «es algo que no puede responderse correctamente sin tener en cuenta (entre otras cosas) lo que el agente puede y no puede hacer realmente en esas circunstancias» (Moya, 2011: 13).

Para ilustrar y justificar esta afirmación propone distintos ejemplos en los dos trabajos mencionados. En Moya (2014) nos ofrece un ejemplo con una serie de variaciones. Se trata de alguien, David, que ve a un niño ahogándose. En la primera versión (Drowning Boy 1), David es un buen nadador, alguien que, si quisiera, podría, muy probablemente salvar al niño. Sin embargo, en lugar de lanzarse al agua para salvarlo, decide gritar pidiendo ayuda.

9 Por razones que no vienen al caso, Moya se centra en la culpabilidad, pero podríamos usar aquí esa misma definición sustituyendo "culpabilidad" por "responsabilidad moral". 
No hay nadie cerca y el niño se ahoga. En la segunda versión (Drowning Boy 2), todo es igual a excepción de que, aunque David no lo sabe, hay una corriente muy fuerte de agua, tal que, aunque David se hubiera lanzado al agua para intentar salvar al niño, no lo habría conseguido. Finalmente en Drowning Boy 3, aunque no hay ninguna corriente de agua, David no sabe nadar (y no es culpable de no saber nadar, su incapacidad no se debe a una falta de interés por aprender sino a una fobia insuperable a las aguas profundas), por lo demás, todo transcurre igual que en las dos versiones anteriores del ejemplo.

Lo que pretende mostrar Moya mediante estos ejemplos es que aquello que supone una alternativa robusta (eximente, dice Moya) en cada caso es distinto. Este es su veredicto, las alternativas robustas son:

-En Drowning Boy 1: Lanzarse al agua, nadar hacia el niño y salvar su vida.

-En Drowning Boy 2: Lanzarse al agua, nadar hacia el niño y tratar honestamente de salvar su vida.

-En Drowning Boy 3: Gritar pidiendo ayuda en un sincero intento de encontrar a alguien que pueda salvarle la vida al niño (Moya 2014: 8).

Y la razón de esto es que, en su opinión, nuestros juicios sobre estos casos son la aplicación de un principio general que formula del siguiente modo:

DBB: Si uno no puede hacer razonablemente más de lo que ha hecho para comportarse de una manera moralmente correcta, no es culpable moralmente por no hacer más (Moya 2014: 7).

En Drowning Boy 1 y 2, según Moya, David es culpable porque no ha hecho todo lo que razonablemente podía hacer, salvar la vida del niño en un caso, intentarlo en el otro, lo que suponía en cada caso una alternativa robusta. En el tercer caso, David no es culpable porque ha hecho todo lo que podía hacer para comportarse de manera moralmente correcta, ha hecho lo que, en su caso, era una alternativa eximente: gritar pidiendo ayuda. Así, pues, Moya pretende defender el PPA mostrando que en todos los casos en que un sujeto es moralmente responsable por una acción existía una alternativa moralmente relevante (robusta, eximente), pero qué es una alternativa de tal tipo depende de las circunstancias de cada caso: según la capacidad o incapacidad de un sujeto y su grado de conocimiento o ignorancia, las alternativas eximentes pueden variar de un contexto a otro, siendo sus estándares más o menos exigentes según los casos.

\section{El caso de Pereboom reexaminado}

Con todas estas consideraciones y bagaje conceptual, Moya pretende demostrar que, a pesar de todo, en el caso de Pereboom, Joe sí que cuenta con una alternativa robusta (y eximente) que es la que justificaría que le atribuyéramos responsabilidad moral, que le culpáramos, y que, claro está, supondría que el caso no fuera una refutación del PPA. Según Moya, Joe es moralmente responsable porque cuenta con una alternativa que, caso de haberla elegido (y aunque él no conozca este hecho), le eximiría de culpa: "recoger evidencia y razones en favor 
de no evadir impuestos y prestarles la debida atención" (Moya, 2011: 16-17). La justificación que se ofrece para esta afirmación es que, de acuerdo con el principio (DBB), Joe no ha hecho todo lo que estaba en su poder para cumplir con sus obligaciones morales. Debía haber tenido en cuenta razones morales y haberles prestado la suficiente atención. Como eso es todo lo que, en sus circunstancias, Joe puede hacer, ello contaría como una alternativa eximente.

Hay, de nuevo aquí, algunas consideraciones que hacer. Ciertamente, culpamos a Joe en tales circunstancias, porque ni tan siquiera ha atendido a dichas razones. En su caso, este hecho es suficiente para que lo culpemos, porque, de haberlo hecho, hubiera evitado evadir impuestos por sí mismo, ya que el mecanismo se hubiera activado. Pero que sea suficiente para atribuirle culpa no significa que lo contrario, que hubiera atendido vivamente a razones morales, sea suficiente para quedar exento de cualquier grado de culpa, sería una condición necesaria pero no tiene por qué ser una condición suficiente. Si lo que Moya denominaba hechos modales - lo que el sujeto puede o no puede hacer en un contexto particular - son relevantes para juzgar si una alternativa es robusta, también lo son las posibilidades contrafácticas, esto es, lo que hubiera podido hacer o dejar de hacer si no hubiera un elemento imposibilitador como es Black. Es verdad que, puesto que Joe tiene implantado el dispositivo en su cerebro, no habría podido decidir no evadir impuestos (ni tampoco habría podido no decidir evadirlos), pues Black le hubiera obligado a decidirlo. Pero también es verdad, que si el dispositivo no se hallara presente, el hecho de considerar con atención razones morales no aseguraría (dado el compromiso indeterminista del ejemplo) su decisión de no hacerlo, de cumplir con su obligación moral; no lo exoneraría completamente. Y si no lo haría en el caso en que no existiera el dispositivo, la imposibilidad de tomar tal decisión por un factor absolutamente ajeno a su voluntad y a su intención y que él ignora, me parece que tampoco debe hacerlo en este caso (en el que el dispositivo está presente), pues decidir no evadir impuestos continúa siendo una posibilidad epistémica para él, (aunque, de hecho, no sea una posibilidad real, él cree que podría, si quisiera, decidir no evadir impuestos). Comparémoslo, si no, con otro caso del propio Moya. Una médico decide (con una decisión sincera) prestar los primeros auxilios a la víctima de un accidente, pero se encuentra con que su maletín esta cerrado con llave (Moya 2011). Intentar abrir el maletín, aunque sólo sea para encontrarse con la sorpresa de que no puede abrirlo, es todo lo que puede hacer en esas circunstancias. En tal caso, la eximiremos de culpa porque ha intentado ayudar, tenemos la garantía y la certeza de que lo ha intentado. No es así en el caso del Joe de Pereboom. Efectivamente no podríamos culparle de su decisión si el dispositivo hubiera intervenido, no podemos culparle por algo que no ha hecho por sí mismo; pero que el dispositivo intervenga meramente porque Joe ha prestado cierto nivel de atención a razones morales (hecho este último que en circunstancias normales no garantizaría que cumpliera con su obligación moral) parece una forma demasiado rápida y fácil de quedar exonerado de toda culpa. Es tener mucha suerte ${ }^{10}$. Al fin y al cabo, atender a razones morales no es "querer algo diferente de lo que realmente quiere", como pediría Pereboom para que una alternativa fuera eximente (Pereboom 2009:112). Que considere razones morales no significa que quiera no evadir impuestos, es aún algo que está muy alejado de la volición, de la decisión voluntaria, y más aún de la acción: podría ser un primer paso en esa dirección, pero que considere dichas razones en su deliberación no significa que quiera no evadir impuestos, sino

10 El mismo Moya (2014: 17 y 19) reconoce que hay involucrado un elemento de suerte. 
sólo que no ha optado aún por evadirlos. Moya había pedido que para que una alternativa sea robusta, el hecho de que el agente la elija o no debe explicar de un modo racional y no sólo causal la mayor o menor culpabilidad del agente por hacer o dejar de hacer lo que ha hecho. Pero me da la sensación que en este caso la consideración de razones morales sólo explicaría de manera causal (y no racional) su supuesta no culpabilidad. En este caso la consideración atenta de razones morales impediría que decidiera evadir impuestos por sí mismo de un modo no muy distinto a cómo lo impediría el hecho de que, mientras delibera, se tomara una taza de café que resultara estar envenenada.

El mismo Pereboom no la considera una alternativa robusta:

¿Cuál es la motivación para pensar que prestar más atención a las razones morales se convierte ahora en una alternativa robusta a decidir evadir impuestos? De acuerdo: es la siguiente mejor acción que le está disponible. Pero no es por sí misma eximente de decidir evadir impuestos, ya que, si no estuviera el mecanismo, incluso si estuviera más atento, podría decidir evadir impuestos y, podemos suponer, sería probable que así lo decidiera. (Pereboom 2012: 304).

Aunque sin saberlo, Joe se hubiera encontrado en una posición de ventaja, respecto de su exención. Sin saberlo, Joe habría deferido su decisión a Black; eso le impediría tomar una decisión (por sí mismo), pero no asegura su inocencia en condiciones normales, no nos asegura que, sin la presencia de Black, hubiera decidido finalmente no evadir impuestos, y esto parece relevante para nuestro juicio moral. Al fin y al cabo, y tal y como mantenía el cuerno indeterminista de la estrategia del dilema: si la consideración de razones morales sólo es un signo fiable, entonces no hay ninguna razón concluyente para pensar que no hubiera podido decidir finalmente de un modo distinto a aquél en que, caso de tener que intervenir, Black le obligaría a decidir (esto es, no hay una razón concluyente para pensar que no hubiera decidido evadir impuestos por sí mismo). Y esta vez la estrategia del dilema se vuelve en contra de los indeterministas.

Culpamos a alguien si no ha puesto de su parte todo lo que en sus circunstancias es necesario para cumplir con su obligación moral. Si A es la acción a la que estamos obligados y B es un medio necesario para tal fin, debemos hacer B. Pero si B no garantiza, en circunstancias normales, la posterior decisión del sujeto de hacer A, y la acción consecuente, entonces en las circunstancias en que no se puede hacer A, ni tampoco decidir hacerlo (por razones completamente ajenas al sujeto y desconocidas por él), la mera ejecución de B no puede ser una alternativa robusta. Hay, pues, algo insatisfactorio en ello.

La única manera en la que tendríamos la garantía de que Joe decidiría no evadir impuestos por la consideración viva y atenta de razones morales es que dicha consideración determinara su decisión posterior, que fuera causalmente suficiente para la misma. En tales circunstancias, ello se convertiría realmente en una alternativa robusta. Esta es la paradoja, que sin determinismo no parece suficientemente robusta, pero el indeteminista no puede aceptar que la decisión esté determinada por aquello que se convertiría, de otro modo, en una alternativa robusta: la consideración de razones morales.

Tal vez tengamos que decir que el Joe de Pereboom sigue siendo culpable porque disponía de alternativas epistémicas justificadas y ha optado (voluntariamente) por la alternativa 
culpable, unas alternativas que se convierten en reales por cuanto comportan actuar por la propia decisión (según la propia voluntad racional) o no hacerlo. Y aunque una de las dos posibilidades no esté bajo su control, es significativo y relevante que él así lo crea. Joe es culpable porque creía que podía actuar de otro modo y, a pesar de ello ha decidido por sí mismo actuar de un modo moralmente reprobable. Es decir, quizá la mera existencia de alternativas epistémicas no sea una condición suficiente para la responsabilidad moral, pero cuando a esto se suma la voluntad racional del sujeto, parece que ya tenemos todo lo necesario. Y al fin y al cabo, si las posibilidades alternativas no son compatibles con el determinismo, las posibilidades epistémicas sí lo son ${ }^{11}$.

No parece, de todos modos que la cuestión esté completamente decidida. En la medida en que la consideración atenta de razones morales tiene relevancia moral, podríamos considerarla aún una alternativa robusta en ese contexto ${ }^{12}$. Para evitar éste problema y otras críticas recibidas, Pereboom rediseñó su caso del modo que sigue (Pereboom 2009:117): ahora Joe es consciente de las razones morales para no evadir impuestos y sensible a ellas, pero estas razones se ven superadas por el propio interés. De modo que, aunque él no lo sabe, es causalmente necesario (aunque no suficiente) para que no evada impuestos "que se imagine, con cierto grado de viveza, siendo castigado, al menos con bastante severidad, por hacerlo, un estado mental que puede generar voluntariamente". Ahora bien, si la cantidad a evadir fuera sustancialmente superior, entonces las razones morales por sí mismas podrían hacer que decidiera no evadir impuestos. Una vez más, para asegurar que decide evadir impuestos, si el dispositivo instalado en su cerebro detectara que está imaginando el castigo, entonces estimularía los centros neuronales oportunos, de tal modo que Joe elegiría inevitablemente evadir impuestos. Pero, de nuevo, Joe no llega a imaginar con viveza tal castigo y decide evadir impuestos sin que el dispositivo tenga que intervenir.

\section{Alternativas muy poco robustas}

Con este nuevo caso, la solución de Moya tiene peores perspectivas. La condición causalmente necesaria (y no suficiente) para que Joe decidiera no evadir impuestos, esto es, imaginar que es castigado, no parece ser algo moralmente mejor que no hacerlo y, como

11 Al fin y al cabo, el determinismo ya es verdadero o no lo es (su verdad no depende de nuestro conocimiento), pero nosotros deliberamos y tomamos decisiones sobre si hacer algo o no hacerlo; sea verdadero o falso de hecho, nosotros creemos que tenemos alternativas. Lo que mantengo, pues, es un compatibilismo entre el determinismo y las alternativas epistémicas. Que nuestra decisión esté determinada no implica que sepamos cuál va a ser ésta antes de deliberar, y si deliberamos es porque creemos que tanto una decisión como su contraria son posibles. Si el determinismo es verdadero sólo es posible hacer A o sólo es posible no hacer A, pero en la medida en que el sujeto no sabe cuál de las dos es en realidad la única metafísicamente posible, tanto a como no-A son posibilidades epistémicas para él. Y esto es así, además, tanto si el sujeto en cuestión es determinista (cree que el determinismo es verdadero) como si no lo es.

12 Justamente, para mostrar que, a pesar de no poder decidir no evadir impuestos, la consideración atenta de razones morales es una alternativa robusta, Moya ofrece una última versión de su Drowning Boy, que llama D y en la cual todo es igual que en Drowning Boy 1, esto es David decide no lanzarse al agua y es culpable. Pero en este caso no podría haber decidido intentar salvar al niño porque, debido a una experiencia traumática en su infancia, que ha olvidado, si David hubiera tomado en serio la posibilidad de lanzarse al agua mediante la seria atención a razones morales, entonces habría sufrido un fuerte ataque de ansiedad y terror, de tal modo que no habría podido tomar tal decisión (Moya 2014:17). 
admite el propio Moya, "dado que él ignoraba que esto era causalmente necesario para que tomara la decisión moral correcta, no es razonable mantener que el tenía que haber imaginado el castigo" (Moya 2011: 18). Pero Moya dispone aún de una réplica:

"Puesto que Joe era consciente de las razones morales en contra de evadir impuestos y sensible a ellas, ¿por qué habríamos de aceptar que Joe no podría haber hecho el esfuerzo de prestar más atención a tales razones y decidir de acuerdo con ellas? Dado que, en esta situación, para que él decidiera en contra de evadir impuestos era necesario que se imaginara siendo castigado, este esfuerzo sería ineficaz a menos que fuera acompañado por tal imaginación, pero el esfuerzo es algo más que él podría hacer para cumplir con sus obligaciones morales. Había, pues, disponible para Joe una "siguiente mejor acción", después de todo, que no ha realizado pero que podría haber hecho" (Moya 2011: 19).

Es claro que tal respuesta merece algunas consideraciones. La única alternativa con relevancia moral de la que dispone Joe es cada vez más débil. Ahora no sólo no tenemos la garantía de que la consideración de razones morales llevarían a nuestro agente a decidir no evadir impuestos, sino que tenemos la certeza de que no lo puede hacer. En todo caso, aquello que lo inclinaría hacia tal decisión sería algo que no tiene relevancia moral: imaginarse castigado por evadir impuestos, no es, ciertamente, la "siguiente mejor acción" (Moya 2014: 23). De hecho, nada se ha dicho de si en el caso que estamos analizando el sujeto ha hecho o no el esfuerzo de considerar razones morales. Quizá lo haya hecho, pero como ello es ineficaz respecto de su decisión de no evadir impuestos, Black, con buen criterio, no ha tenido que intervenir. ¿Qué relevancia moral tiene entonces que las haya considerado? ¿No seguimos culpando del mismo modo a Joe?

No está claro, pues, si el esfuerzo que está pidiendo Moya es el mero considerar razones morales (con toda la atención que se quiera), o el de "prestar más atención a tales razones y decidir de acuerdo con ellas". Pero, en el ejemplo de Pereboom, la segunda parte (decidir de acuerdo con ellas) no es posible, no es una alternativa real y Moya es consciente de ello. A pesar de todo, mantiene, debería haberlas considerado. Sí, debería haberlas considerado, pero difícilmente ello sería un atenuante si finalmente, como es el caso, decide evadir impuestos por sí mismo. Así, la única solución que parece quedar abierta a Moya es negarle a Joe su carácter de agente moral responsable, considerar que si Joe no es capaz de decidir no evadir impuestos en esa ocasión - en la que la suma a evadir no es muy elevada - por la mera consideración de razones morales, entonces no es un agente moralmente responsable, un agente apto para que le sea atribuida responsabilidad moral ${ }^{13}$. Pero si aceptamos esto, ello comportaría que los agentes moralmente malvados - puesto que son los menos susceptibles de comportarse según razones morales, hasta el punto de que en muchas circunstancias y contextos éstas son ineficaces a la hora de llevarles a actuar - no serían agentes moralmente responsables. Una conclusión difícil de aceptar.

13 Eso o negar que se pueda aceptar "que sea literalmente imposible causalmente para él tomar la decisión moralmente correcta sobre la única base de sus razones morales presentes" (Moya 2014: 24). Tal estipulación hace, en su opinión, que el ejemplo no sea psicológicamente creíble. 
De hecho no parece implausible en absoluto que la mera consideración de razones morales sea causalmente insuficiente para llevar a alguien a decidir no evadir impuestos si la cantidad no es muy elevada, y que sólo decidiría no hacerlo por (o sumándole) el temor a ser castigado. Podemos perfectamente imaginar un sujeto tal que, únicamente si cambiamos las circunstancias antecedentes, esto es si incrementamos sustancialmente la cantidad a defraudar, se vea movido a decidir no evadir impuestos, pero que en las circunstancias presentes tales consideraciones sean causalmente ineficaces si no van unidas a la imaginación del castigo. No veo en esto ninguna implausibilidad psicológica.

Si en el caso anterior de Pereboom ya teníamos serias dudas para considerar robusta la única alternativa de que disponía el agente, ahora, obviamente, tales dudas se incrementan en sumo grado. El atenuante que ahora puede suponer la consideración de razones morales, en la medida que sabemos que no puede producir en ningún caso la decisión eximente, sólo puede ser muy débil, si es que es atenuante en alguna medida.

\section{Conclusión: las alternativas meramente epistémicas como alternativas robustas}

Sin embargo, seguimos culpando a Joe porque disponía de la alternativa epistémica justificada de no evadir impuestos. Él creía que podía tomar tal decisión, no tenía ninguna razón para pensar que no podía tomarla, y no ha hecho nada de su parte o ha hecho demasiado poco. La existencia de alternativas epistémicas, es, una vez más en tal contexto (donde el agente ha hecho algo intencionalmente y creía que podía no hacerlo), condición suficiente de su responsabilidad moral. Se trata de alternativas robustas, porque determinan nuestro juicio moral.

Así que, llegamos a la siguiente conclusión. Las alternativas epistémicas justificadas, las meras alternativas epistémicas justificadas, son todas las alternativas que se necesitan para la atribución de responsabilidad moral, son, ellas mismas, alternativas robustas. Las alternativas epistémicas justificadas (junto con la decisión racional voluntaria) son condición suficiente de la responsabilidad moral, puesto que, aunque Moya tuviera razón y el considerar razones morales fuera una alternativa robusta real (y no sólo epistémica: evadir impuestos por uno mismo o no), es la alternativa epistémica de no evadir impuestos la que la genera. Así, si siempre existe una alternativa real, tal como inclinarse hacia una determinada decisión, es la alternativa epistemica de decidir de ese modo la que la garantiza. Las alternativas epistémicas, pues, son condición suficiente (junto con las otras condiciones mencionadas) de la responsabilidad moral, porque o bien se bastan en sí mismas o son garantía de la existencia de una alternativa real.

Nuestra conclusión, entonces es que el PPA debería ser sustituido por el principio de posibilidades epistémicas alternativas, PPEA: un sujeto es moralmente responsable de su decisión y de su acción consiguiente si éstas son el resultado de la voluntad racional del sujeto, y el sujeto cree que podría decidir o actuar de otro modo (o al menos cree que podría decidir evitar actuar del modo que lo hace).

Obsérvese que este principio (PPEA), a diferencia del PPA, mantiene que junto con la voluntariedad y el control racional por parte del agente, las alternativas epistémicas son condición suficiente de la responsabilidad moral. No se compromete en cambio con que sean necesarias. Lo que dice el principio es que dadas las condiciones de voluntariedad y sensibi- 
lidad a razones, basta con la existencia de posibilidades epistémicas para que el agente sea moralmente responsable. El principio, sin embargo no exige que existan tales posibilidades para que haya responsabilidad moral. Quizá sea posible ser moralmente responsable de una acción para la que se sabe (o se cree justificadamente) que no hay alternativa, respecto de esto, nada dice el PPEA.

\section{Referencias}

DAVIDSON, D. (1973), "Freedom to Act", en Honderich (1973).

FISCHER, J. M. (1994), The Metaphysics of Free Will, Oxford, Blackwell.

FISCHER, J. M., KANE, R., PEREBOOM, D. and VARGAS, M (2007), Four Views on Free Will, Oxford, Blacwell.

FRANKFURT, H. G. (1969), “Alternate Possibilities and Moral Responsibility”, The Journal of Philosophy, 66: 829-839.

GINET, C. (1996), "In Defense of the Principle of Alternative Possibilities: Why I Don't Find Frankfurt's Argument Convincing”, Philosophical Perspectives, 10: 403-417.

HONDERICH, T. (ed.) (1973), Essays on Freedom of Action, Oxford, Routledge.

KANE, R. (1985), Free Will and Values, Albany, State University of New York Press.

LOCKE, J. (1689/1956), Ensayo sobre el entendimiento humano, traducción de Edmundo O’Gorman, México, F.C.E.

MOYA, C. J. (2011), "On the Very Idea of a Robust Alternative”, Crítica. Revista Hispanoamericana de Filosofía, 43: 3-26.

MOYA, C. J. (2014), "Doing One's Best, Alternative Possibilities, and Blameworthiness", Crítica. Revista Hispanoamericana de Filosofía, 46: 3-26.

NAYLOR, M.B. (1984), "Frankfurt and the Principle of Alternate Possibilities", Philosophical Studies,46: 249258.

PEREBOOM, D. (2009), "Further Thoughts about a Frankfurt-Style Argument", Philosophical Explorations, 12: 109-118.

PEREBOOM, D. (2012), "Frankfurt Cases, Derivative Responsibility, and the Timing Objection", Philosophical Issues, 22: 298-315.

VAN INWAGEN, Peter (1983), An Essay on Free Will, Oxford, Clarendon Press.

VARGAS, M. (2007), “Revisionism”, en Fischer, J.M., Kane, R., Pereboom, D. and Vargas, M (2007): 126-165.

WARFIELD, T. A. (2000), "Causal Determinism and Freedom Are Incompatible: A New Argument for Incompatibilism”, Philosophical Perspectives 14: 167-180.

WIDERKER, D. (1995), "Libertarianism and Frankfurt's Attack on the Principle of Alternative Possibilities", Philosophical Review: 104: 247-261.

WIDERKER, D. (2006) "Libertarianism and the Philosophical Significance of Frankfurt Scenarios", The Journal of Philosophy, 103,: 163-87.

WIDERKER, D. (2009), "A Defense of Frankfurt-friendly Libertarianism", Philosophical Explorations 12: 87-108. 